\title{
Targeting T-cell immunometabolism during transplantation
}

\author{
SHADAB KAZMI ${ }^{1}$, Mohammad Khan ${ }^{1}$, Talal Shamma ${ }^{1}$, Abdullah Altuhami ${ }^{1}$, Abdallah \\ Assiri $^{1}$, and Dieter Broering ${ }^{1}$ \\ ${ }^{1}$ King Faisal Specialist Hospital and Research Center
}

November 30, 2020

\begin{abstract}
The balance between the immune system and its metabolism is becoming an effective therapeutic alternative in various inflammatory diseases, including organ transplantation. The interaction between the immune and metabolic pathways play a critical role in dictating disease pathology and progression, and the differences in the bioenergetic demands between immune cells enable them to differentiate into effector and regulatory cells. Recent studies have suggested that changes in intracellular metabolic programs control $\mathrm{T}$ cell activation, proliferation and differentiation into $\mathrm{T}$ effector (Teffs) or $\mathrm{T}$ regulatory cells (Tregs), and metabolic differences between Tregs and Teffs can shift the balance toward a more specific immune tolerance in organ rejection. Pharmacological targeting of $\mathrm{T}$ cells metabolism affects the balance between effector and regulatory function of $\mathrm{T}$ cells. This therapeutic modulation are of great interest in cancer, autoimmunity, and organ transplantation. In this review, we discuss major metabolic pathways that influence the activation, proliferation and differentiation of Tregs, and also special emphasis on liver kinase B1 (LKB1) pathway that provide stability of Tregs. We also highlight how Tregs metabolic regulome and LKB1 signaling pathway relationship contribute to rescue organ transplants from associated injuries and chronic rejection.
\end{abstract}

\section{BACKGROUND:}

The metabolic heterogeneity of immune cells provide an opportunity for different immune cells to differentiate into effector or regulatory cells. Targeting the metabolism of immune cells with metabolic inhibitors is a new approach in immunosuppression. Therefore, selective inhibition of basic metabolic pathways, which are necessary for all healthy cell survival, can still show selectivity toward immune cells. It all depends on the degree of reliance on immune cells on those pathways. The delicately balanced interplay between protective immunity and inflammatory conditions is critical for the activation/differentiation of various immune cells. On encounter with antigen and receiving appropriate co-stimulatory signals, naïve $\mathrm{T}$ cells activate downstream metabolic reprogramming for rapid cell growth and proliferation to fuel their specific effector functions. Immunometabolism provides a greater room for a therapeutic window in transplantation settings and autoimmune disease treatment. Furthermore, LKB1 is an important upstream kinase that strengthen the stability of Tregs, and their signaling can be co-regulated to enhance the stability of Tregs. Notably, LKB1 is a metabolic regulator that connects cellular metabolism to immune cell functions. Recent work has demonstrated that modulating Treg function by targeting metabolic pathways and LKB1 signaling in models of transplantation and inflammatory diseases do not cause any visible toxicity, which advocates this strategy as a new therapeutic intervention for treating inflammatory diseases.

\section{IMMUNOMETABOLISM:}

Immunometabolism has emerged as one of the exciting areas in the field of translational research. More recently, the role of cellular metabolism in the development and function of immune cells in healthy and diseased individuals has been described in diseases such as cancer, autoimmunity, and organ transplantation. 
Metabolic reprogramming of distinct $\mathrm{T}$ cell subsets is the center for appropriate $\mathrm{T}$ cell differentiation and function [1-3]. In this line, T cells use many different metabolic pathways to generate ATP for their survival and boost numerous molecular biosynthetic precursors production for their proper proliferation and differentiation [4]. These metabolic pathways entail diverse end products, but they are interconnected on shared fuel ground. For example, fatty acid synthesis pathway is dependent on the availability of intermediate products from the glycolysis and tricarboxylic acid (TCA) cycle metabolism. Thus, metabolic regulome of immune cells is a key factor in the control of immune responses.

CELL ENERGY: Cells mostly use glucose as the source of energy; however, few cells use fatty acids or amino acids as the source of energy depending on their energetic demands [5-7]. Typically, the cell allows the uptake of these energy-rich molecules through glycolysis and enter the TCA cycle, and the electron transport chain (ETC) to produce ATP [8, 9]. Glycolysis involves the uptake of extracellular glucose into cells through the glucose transporter 1 (GLUT1) and subsequent conversion into pyruvate along with numerous other products inside the cytosol [10]. This glucose metabolism is a relatively inefficient pathway in terms of cellular ATP production; however, it provides a way to maintain redox balance inside the cell (to reduce $\mathrm{NAD}^{+}$into NADH), which is subsequently used by various enzymes as a cofactor and enables the diversion of metabolic intermediates to biosynthetic growth pathways to support anabolic growth [11, 12]. The TCA cycle occurs in the mitochondrial matrix and it uses pyruvate as a substrate [13], which is converted into acetyl coenzyme A $(\mathrm{CoA})$ to generate energy-rich electron carriers NADH and FADH2. These electron carriers transport electrons through oxidative phosphorylation (OXPHOS) to the inner mitochondrial membrane into the ETC to generate a hydrogen gradient required for the synthesis of ATP [14]. Besides, the fatty acid oxidation (FAO) pathway, which is a key catabolic pathway for energy production, involves the mitochondrial conversion of fatty acids into several products that are subsequently used by the cell to generate energy using fatty acids instead of glucose [7]. Activation of the fatty acid is the first step of FAO to occur in the cytosol that generates acetyl-CoA, which then enters the TCA to generate electron carriers, and then enter the ETC to generate energy [15]. In the context of immunity, a high rate of glucose metabolism has been found in lipopolysaccharide (LPS)-activated macrophages and dendritic cells (DCs), activated natural killer (NK) cells, activated effector T cells, and activated B cells [16-20]. Indeed, this upregulation of glycolysis in immune cells can be considered a hallmark metabolic change for immune cells that are undergoing rapid activation, explicitly in response to stimulation of pattern recognition receptor (PRR), cytokine receptors, or antigen receptors. Aerobic glycolysis has been associated with inflammatory and rapidly proliferating immune cells; however, dependency on FAO has been observed in many non-inflammatory immune cells and show raised cellular lifespans, including M2 macrophages, Tregs, and memory $\mathrm{T}$ cells [7, 21-23]. In contrast to FAO, fatty acid synthesis regulates the generation and activation of pro-inflammatory immune cells. Therefore, inflammatory signal leads to fatty acid synthesis of inflammatory immune cells, whereas tolerogenic stimuli drive FAO of non-immune cells. Moreover, activated T cells also use few amino acids, especially glutamine metabolites to generate $\alpha$-ketoglutarate as a substrate of the TCA cycle to generate energy, and most importantly, through the mTOR pathway for sensing amino acid levels inside the cell and for nucleotide synthesis [24-26].

T CELL ENERGY: The metabolic pathways described above play a significant role in determining $\mathrm{T}$ cell functional outcomes. In the primary lymphoid organs, undifferentiated naïve $\mathrm{T}$ cells rely on mitochondrial OXPHOS for energy, as well as on extrinsic cell signals such as IL-7 to maintain energy for immune surveillance before activation [3]. Upon activation in the secondary lymphoid organs, a substantial change in the metabolism of naïve $\mathrm{T}$ cells allows them to differentiate into various Teff cells. Teffs such as activated $\mathrm{CD}^{+}$and $\mathrm{CD}^{+}{ }^{+} \mathrm{T}$ cells depend on aerobic glycolysis for their bioenergetic demands due to the need for a rapid immune response [27]. In contrast to Teffs, Tregs use lipid metabolism instead of glycolysis [28] and mainly rely on OXPHOS generating long-lasting energy. Memory T cells (Tmem) also depend on FAO and OXPHOS for energy and have a high mitochondrial mass to generate energy rapidly in secondary exposure to the same antigen to execute rapid response. Notably, FAO is essential to maintain mitochondrial spare respiratory capacity (SRC) in Tmem cells. SRC is a functional parameter of mitochondria that measure 
maximum capacity of a cell to consume oxygen during stress [29]. Therefore, Treg and Tmem mainly rely on OXPHOS and FAO, for their energy demand, and express low levels of Glut1 and high rate of lipid oxidation that also further strengthen their dependency on FAO [28, 30]. As stated earlier, Teffs (Th1, Th2, and Th17) cells need glycolysis and to the lower extent rely on OXPHOS for their energy requirements and express high levels of Glut1 that underpins their high glycolytic nature [31]. Subsequent research studies demonstrated that Tregs have elevated expression of FAO related genes such as AMP-activated protein kinase (AMPK) compared to Th17 cells [32]. Previous research suggests that long chain FAO impart Tregs and Tmem cells survival. This conception is mainly due to inhibition of carnitine palmitoyltransferase 1A (CPT1A) with the drug etomoxir. CPT1A is the rate-limiting enzyme for long chain FAO and facilitate transport of long chain fatty acids into mitochondria for its $\beta$-oxidation. In turn, further findings show that Treg cells and Tmem cells utilize various pathways to transport fatty acids and use short- or medium-chain fatty acids whose translocation is not dependent on CPT1A $[33,34]$. Recent research shed new light that, engagement of the inhibitory programmed cell death 1 (PD1) receptor on T cells with its ligand PD-L1 resulted in enhanced expression of CPT1A and elevated FAO. Hence, this engagement of PD1 prevents effector cell development [35]. Notably, PD1 is a crucial regulator of immune homeostasis, and its engagement increases the longevity of $\mathrm{T}$ cells in an oxidative environment. In marked contrast, cytotoxic $\mathrm{T}$ lymphocyte antigen 4 (CTLA4) inhibits glycolysis without augmenting CPT1A and FAO. Under normal conditions, Teffs cells downregulate FAO during their activation process and enhance fatty acid synthesis for their growth [27]. This downregulation of FAO by Teffs is attributed to Teffs functional inhibition and enhanced immune tolerance by FAO. Moreover, the glycolytic-lipogenic pathway and glutamine metabolism are used to fuel mitochondrial OXPHOS through the TCA cycle as an alternative input for the TCA cycle to support ATP production. It is mainly associated with Teffs differentiation through the mTOR pathway [36], which is the central player of CD4 T cells differentiation [37]. These different metabolic programs are tightly regulated to facilitate the energy production required for each distinct $\mathrm{T}$ cell subset. Glycolysis, OXPHOS, and glutaminolysis, are intertwined and properly managed to fulfill the bioenergetics demand of immune cells for proper proliferation and differentiation (Figure 1). Collectively, these studies indicate key roles of metabolic programming in determining T-cell fate and function along with other signals such as the strength of TCR stimulation, inflammatory cytokines and transcriptional factors [38, 39].

REGULATORY T CELLS AND EFFECTOR T CELLS METABOLISM: Tregs are a subset of $\mathrm{CD} 4^{+} \mathrm{T}$ cells that play a vital role in maintaining immunological homeostasis, preventing autoimmunity, and graft rejection due to their potent immunosuppressive and reparative activities [40-44]. To accomplish these multiple roles in control of the disproportionate inflammatory response and tissue injury Treg cells express various effector molecules for their activation and stability [45]. Tregs have two main subsets, thymus-derived natural Treg (nTreg) and peripherally (induced) derived Treg (iTreg) cells. iTregs are generated at peripheral sites from naïve $\mathrm{T}$ cells after TCR stimulation in the presence of TGF- $\beta$, or they can arise from conventional effector T cells [46].

In transplantation, donor-specific tolerance is a crucial parameter for a high survival rate, which could be achieved by increasing Tregs or by suppressing donor reactive $\mathrm{T}$ cells. Therefore, the differences in the metabolism between effector and regulatory $\mathrm{T}$ cells make immuonometabolism a promising therapeutic intervention that could allow for a more specific immune tolerance in the field of transplantation [47]. Numerous preclinical models of autoimmune diseases and transplantation have shown that Treg cells maintain peripheral tolerance after tissue injury and exposure to intracellular antigens or alloantigen [48]. Various $\mathrm{T}$ cells adjust their metabolic programming to meet the energetic demands necessary for their cellular functions. As discussed in the earlier section, for respiration, a naïve $\mathrm{T}$ cell mainly depends on OXPHOS, and later on, upon antigen encounter, $\mathrm{T}$ cell activates aerobic glycolysis necessary for effector cytokine production $[47,49]$. Activated $\mathrm{T}$ cells use aerobic glycolysis as a fast generating energetic mechanism to satisfy the immediate energetic demands for proper proliferation and differentiation. This use of aerobic glycolysis by $\mathrm{T}$ cells was first described by Otto Warburg in cancer cells and hence is known as the Warburg effect [50]. The two main subsets of $\mathrm{T}$ cells $\left(\mathrm{CD} 4^{+}\right.$and $\mathrm{CD} 8^{+} \mathrm{T}$ cells) show many similarities in their activation. Both activated subsets enhance their dependency on glycolysis and increase Glut1 expression for glucose uptake; 
however, they have different metabolic profiles. CD8 ${ }^{+} \mathrm{T}$ cells rely on glycolysis less than $\mathrm{CD} 4^{+} \mathrm{T}$ cells due to diminished glycolytic enzyme expression $[51,52]$. Alternatively, $\mathrm{CD} 4^{+} \mathrm{T}$ cells display a marked increase in mitochondrial mass as compared to $\mathrm{CD} 8^{+} \mathrm{T}$ cells, while $\mathrm{CD} 8^{+} \mathrm{T}$ cells show greater dependency on OXPHOS for cytokine production [53]. Activated CD4 ${ }^{+} \mathrm{T}$ cells differentiate into Teffs (Th1, Th2, or Th17 cells) by triggering different metabolic pathways downstream of the TCR and by the availability of essential metabolites.

Tregs have different metabolic profiles compared to $\mathrm{CD}^{+} \mathrm{T}$ effector cells. Tregs utilize FAO for their differentiation, unlike Teffs cells $[54,55]$. Furthermore, Tregs depend on OXPHOS for proliferation and steady, long-lasting suppressive functions as seen in Tmems [23, 28]. In Tregs, FOXP3 expression use fatty acids, upregulate ETC, and ATP generation through OXPHOS. Moreover, FOXP3 initiates increased fatty acid $\beta$-oxidation, which results in the selective protection of FOXP ${ }^{+}$cells from fatty acid-induced cell death [56]. This phenomenon is crucial to provide a key target for modulating Treg function and selection in clinical settings. Recent findings have identified an inverse relation for the metabolic phenotype in mouse Tregs and human Tregs. In murine models, iTregs displayed low glycolytic rate, whereas, human iTregs, preferentially use glycolysis for their development and function because of enolase- 1 suppresses the transcription of the exon 2-containing FOXP3 splicing variant unless engaged in glycolysis [32, 57]. This splicing variant is important for Treg mediated suppression.

The metabolic profiling of $\mathrm{T}$ cell subsets also reveals their dependency on glycolysis or OXPHOS. The enzyme pyruvate dehydrogenase (PDH) has been found as a central node in the programming of $\mathrm{T}$ cells to use either glycolytic or oxidative metabolism for their differentiation. Indeed, pyruvate metabolism plays a crucial role in Teffs and Tregs conversion. The enzymatic activity of PDH is inhibited by PDH kinases (PDHKs). PDHKs are highly expressed on Th17 cells, but not on Th1 cells and at low levels in Tregs under the influence of hypoxia inducible factor 1- $\alpha$ (HIF1- $\alpha)$ [2, 58-60]. Inhibiting PDHK1 with specific inhibitors such as dichloroacetate (Table 1), which promotes oxidative phosphorylation selectively suppresses Th17 cells therefore favors Tregs generation [61]. This suppression causes the production of reactive oxygen species (ROS), and treating with ROS scavengers such as N-acetyl cysteine (NAC) restore Th17 cell generation [2].

Lipids, especially short-chain fatty acids, are a preferential source of acetyl groups for histone acetylation and epigenetic reprogramming. FAO reprogram cellular metabolism and this oxidation is also the primary source of lipid-derived acetyl-CoA [62]. A recent finding shows that post-translational modifications such as acetylation, ubiquitination, and phosphorylation control FOXP3 expression, therefore, FAO supports the immunosuppressive function of Treg by regulating histone acetylation in the FOXP3 locus [63, 64] which stabilize the FOXP3 expression [65, 66] (Figure 1).

In addition to glucose and fatty acids, cells also utilize glutamine and leucine amino acids, which play a vital role in Teff's differentiation. CD4 ${ }^{+} \mathrm{T}$ cells deprived of glutamine differentiate into Tregs in- vitro condition [67]; however, iTregs show less dependency on amino acids for their energy requirements [68]. Glutamine metabolism involves the influx of glutamine in the TCA-cycle in the form of $\alpha$-ketoglutarate. This intermediate encourages Th1 cell differentiation by enhancing the expression of the inflammatory transcription factor T-bet $[28,68]$. The deletion of neutral-amino-acid transporter genes (Slc7a5 and Slc1a5 (also known as ASCT2) reduce glutamine uptake, glucose metabolism, and overall Teffs differentiation, however, it does not affect the generation of iTregs $[69,70]$. These metabolic variances show that Tregs have less dependency on amino acid metabolism for producing energy compared to Teffs.

Similarly, the metabolic by-products of tryptophan, such as kynurenine, triggers iTreg proliferation by its binding to the aryl hydrocarbon receptor [71, 72]. However, iTregs in the absence of tryptophan can activate the amino-acid-starvation sensor GCN2 (general control nonderepressible-2) kinase that inhibits Th17 cell differentiation $[73,74]$ and supports Treg stability. It has been reported that Tregs upregulate aminoacid-consuming enzymes including ARG1 (arginase 1), HDC (histidine decarboxylase), TDH (threonine dehydrogenase), and IL4I1 (interleukin-4 induced 1) in skin graft as compared to fresh skin to induce tolerance. These findings suggest that Tregs can modulate the concentration of essential amino acids and their catabolic products in the intrinsic cell milieu through the activation of amino acid starvation sensors [75]. In 
this way, Tregs trigger suppression through amino acid starvation and limit the pathology during the normal immune response.

Thus far, we have discussed activation and differentiation of $\mathrm{T}$ cells that lead to metabolic programming. Likewise, in the hostile envoirnment such as in the case of tumor, cellular metabolism reshape in ways that are central to $\mathrm{T}$ cells exhausation and at the same time Tregs sustainability. The tumor microenvironment poses metabolic hurdles such as hypoxia, low glucose, and high lactate concentration, meanwhile tumor cells require immune tolerance to evade host immunity. The upregulation of lactate impairs effector, and cytotoxic $\mathrm{T}$ cells function through LDH-mediated NAD depletion. However, Tregs resist this environment through FOXP3 expression [76], which acts as an intrinsic metabolic regulator that suppresses mTOR- and Mycsignaling pathways that activate- glycolysis while enhancing OXPHOS and NAD+/NADH generation and levels of FAO. These metabolic adaptations in Tregs allow them to sustain in severe inflammatory microenvironments without affecting their function, survival, and suppression, which is vital to maintain peripheral immune tolerance $[56,76,77]$. Additionally, tumor-infiltrating Tregs frequently display substantially upregulated expressions of co-inhibitory receptors, such as $\mathrm{T}$ cell immunoreceptor with immunoglobulin and ITIM domains (TIGIT), lymphocyte activation gene 3 (LAG3), neuropilin 1 (NRP1), PD1, and CTLA4, to sustain stable FOXP3 activity, FOXO1 nuclear localization and higher levels of suppressive function [78].

Contrary to the tumor microenvironment, in other inflammatory conditions, such as in transplantation settings or autoimmunity, Tregs lose their suppressive function and convert into effector cells that produce pro-inflammatory cytokines (IFN $\gamma$, IL-17, IL-4) and have increased expression of associated master regulator transcription factors (such as T-bet, IRF4, and ROR rt, or IRF4 and GATA3). This functional impairment of Tregs causes loss in FOXP3 expression as well as hyperactivation of the inflammatory PI3K pathway [77].

IMMUNOMETABOLIC REGULATION OF TREGS: Multiple signaling networks, for instance, PI3K/Akt, mTOR-HIF-1 $\alpha$ axis, and LKB1-AMPK pathways coordinate after immune cells are stimulated to mediate metabolic reprogramming. Here we focus on LKB1 signaling, in which LKB1 protein that restrains the activation and pro-inflammatory function of Teffs. LKB1 is a bioenergetic sensor that is expressed by the serine-threonine kinase 11 (STK11) gene and regulates cell polarity and function [79- 82]. It is an important upstream kinase that phosphorylates AMPK $\alpha$ (AMPK is heterotrimer having catalytic subunit (AMPK $\alpha$ ) and regulatory subunit (AMPK $\beta$ and AMPK $\gamma$ ) ) that contributes to T cell differentiation and function and for maintaining functional fitness of Tregs in conditions of energy stress. [83]. Maclver et al. have reported that LKB1-AMPK signaling negatively regulates $\mathrm{T}$ cell effector function through the regulation of mTOR activity [84]. Of note, later studies have reported that the LKB1 signaling pathway promotes OXPHOS and FAO to maintain Treg survival and function. Furthermore, Nanhai et al. reported that LKB1 is crucial for maintaining cellular metabolism and energy homeostasis in Tregs and this metabolic phenotype is independent of AMPK and mTOR signaling pathways [85, 86]. To proof this, Nanhai et al. and others have deleted the LKB1 gene in Treg and catalytic subunit deletion of AMPK in Tregs. They found that LKB1 deletion resulted in loss of Tregs number and function and leads to impaired cellular metabolism in Tregs and uncontrolled immune activation. Contrary to this, the catalytic subunit deletion of AMPK in Tregs does not cause any abnormalities in the murine model. These findings are similar to those mice having mutations or deletion of FOXP3, where FOXP3 deletion resulted in loss of Tregs number. These studies further advocate the importance of LKB1 in Treg cell metabolism [80, 85-87].

LKB1 stabilizes FOXP3 expression in Tregs that maintain immunological self-tolerance and homeostasis [80]. Di Wu et al. have reported that LKB1 governs Treg survival and its lineage identity [80]. T cell's specific deletion of LKB1 causes a halt in Treg regulatory function that leads to impaired immune responses [80, 87]. Moreover, LKB1 deficient Tregs have been characterized by defective mitochondria, compromised OXPHOS, depleted cellular ATP, and altered cellular metabolism pathways [80]. Treg-specific deletion of LKB1 leads to the development of fatal autoimmune inflammation [80,88], and causes disrupted Treg survival and reduced mitochondrial mass, its membrane potential, and increased the generation of ROS [85, 86, 89]. Mechanistically, LKB1 deficiency causes the diminished release of intracellular ATP and induces aberrant expression of immune regulatory molecules such as PD-1, and TNF receptor GITR, and OX40 [85, 
90-93]. Yang $\mathrm{K}$ et al. in his classic work has been reported that LKB1 function in Tregs does not depend on AMPK signaling or the mTORC1-HIF- $1 \alpha$ axis as described earlier, however, it depends on LKB1- $\beta$-catenin signaling to regulate PD-1 and TNF receptor proteins such as GITR and OX40 expression on Treg cell [9, $21,85]$. $\beta$-catenin is a key mediator of Wnt signaling and suppresses the aberrant expression of PD-1 and GITR in Treg cells. LKB1 deficient Treg cells show the degradation of $\beta$-catenin. Briefly, these findings indicate the role of LKB1- $\beta$-catenin signaling in the control of Th2 response by modulating PD1 and other Treg signature molecules [85, 94].

It has been reported in many clinical studies that LKB1 is mutated in $20 \%-30 \%$ of NSCLC (Non-smallcell lung carcinoma) patients, which causes enhanced sensitivity to metabolic inhibitors or stress-induced mitochondrial dysfunction [95]. Further, Yang et al. have reported that Tregs need the LKB1 gene to manage their metabolic and immunological homeostasis function, and deficiency of LKB1 resulted in the apoptotic and functional exhaustion of Tregs [85]. Xiuhua $\mathrm{Su}$ et al. have demonstrated that Tregs from acute graftversus- host disease (aGVHD) patients show an exhausted phenotype, which is characterized by the unstable FOXP3 expression, diminished suppressive functions, defective migration capacity, increased apoptosis, and downregulation of LKB1 expression [96]. In addition to maintaining suppressive activity, LKB1 maintain FOXP3 stability in Tregs by demethylation of conserved non-coding sequences (CNS2) at the FOXP3 locus [75] through the activation of signal transducer and activator of transcription 4 (STAT-4), and partially through suppressing nuclear factor- $\varkappa \mathrm{B}(\mathrm{NF}-\varkappa \mathrm{B})$ signaling [97]. Meanwhile, LKB1 promotes Treg suppressor function by increasing the expression of various immunosuppressive genes by enhancing transforming growth factor- $\beta$ (TGF- $\beta$ ) signaling. The deletion of TGF- $\beta$ leads to autoimmune glomerulonephritis and impaired Treg activity [98]. Thus, TGF- $\beta$ pathway is vital for nTreg and iTreg development $[94,95]$.

Treg cells exert their suppressive function in several different ways. One mechanism is through the LKB1 pathway that activates the mevalonate pathway, which is crucial for Treg functional fitness and stability [85]. This pathway also activates its metabolite geranyl pyrophosphate (GGPP), which phosphorylates STAT-5 via IL-2 signaling and subsequently support Treg function and lineage stability. Activation of mevalonate genes by LKB1 is required for Treg proliferation and, thereby suppressing the interferon-gamma (IFNy) and interleukin-17A (IL-17A) expression, which is independent of the AMPK signaling [85]. Furthermore, LKB1 induced the mevalonate pathway was also found to maintain intracellular cholesterol homeostasis [87, 91]. LKB1 signaling is key for Tregs to maintain their metabolic and immunological balance to curb apoptotic and functional exhaustion, thereby reinforcing homeostatic control of Tregs. Altogether, these preclinical investigations highlighted that LKB1 is a primary regulator of lipid metabolism in Tregs, which play a regulatory role in modulating Tregs suppressive activity and maintaining the phase of immunotolerance (Figure 2).

IMMUNOMETABOISM AS A THERAPEUTIC TARGET IN TRANSPLANTATION: Organ transplantation is the last therapeutic approach used to treat end-stage organ failure. Current therapeutic approaches for organ rejection target overall immune suppression, which is associated with severe side effects, cancer, and mortality. However, targeting a more specific immune suppression has become more favorable in clinical settings to treat transplant rejection. The long term survival of transplanted graft is limited by surgical trauma and ischemaic reperfusion injury (IRI). These unavoidable events trigger innate immune cell activation that eventually promote sterile inflammation. This prolong inflammation causes immunometabolic rewiring especially after ischemia and IRI to fulfill oxgen demand. Ischemia or Hypoxia reduces OXPHOS and hence, induce tissue reliance on aerobic glycolysis [99]. This event enhance the production of proinflammatory cytokine that subsequently leads to $\mathrm{T}$ cell activation. In fact,the metabolic requirements of Teffs and Tregs and the conclusive role of Teffs/Tregs ratio have been crucial parameters to decide the fate of the transplanted organ. These metabolic requirements could be used as a key tool in specific therapeutic approaches to contain graft associated microvascular injuries and induce specific immune tolerance. In alloimmune inflammation post-transplantation, donor immune cells rely less on lipid metabolism for their energy requirements, whereas the recipient cell depends on aerobic glycolysis [74, 98]. Specifically, CD4 ${ }^{+} \mathrm{T}$ cell activation in solid organ transplantation more closely resembles the classical metabolic reprogramming that is seen during normal $\mathrm{T}$ cell activation [1]. 
It has been reported that inhibiting glycolysis by metabolic inhibitors such as 2DG (2-Deoxy-D-glucose) and metformin (inhibits complex I of ETC) (Table 1) can considerably decrease [74] the glycolysis of an activated Teff and their cytokine production [101-103]. These two drugs and 6-Diazo-5-oxo-L-norleucine (DON) (Table 1), a glutamine analogue, has been reported to abolish the immune response. In transplant settings, combined therapy such as 2DG, metformin, and DON could suppress the proliferation of pathogenic Teff cells and promote the generation of antigen-specific Tregs; however, combination therapy does not cause the global inhibition of immune responses; instead, it selectively inhibits effector responses while promoting Treg responses based on differential metabolism of immune cells resulted in a specific immune suppression [103]. This combinational therapy targets explicitly allogeneic Teffs that cause graft rejection and keeps other immune cells and healthy tissues relatively unharmed. This combined therapy reduces skin and heart allografts rejection through the inhibition of allogeneic Teffs, and by stimulating Treg proliferation and activation [103-105]. Furthermore, metformin (AMPK agonist) and Soraphen A (acetyl-CoA carboxylase inhibitors) (Table 1) enhance pTreg differentiation by inhibiting allogeneic Teffs in autoimmune mouse models of allergic asthma and experimental allergic encephalomyelitis respectively (Figure 3) [60, 106-109].

Various strategies have been implemented to increase the numbers and potency of Tregs in vivo to reduce the severity of graft rejection [110]. Besides targeting metabolic pathways, some studies have also highlighted that the augmentation of the LKB1 pathway is immensely beneficial in reducing graft rejection, and strategies that could activate the LKB1 pathway might be employed as a future treatment in transplantation settings [96]. Some strategies such as overexpressing LKB1 in lentivirus vectors and then use these vectors to create genetically reprogrammed Tregs and then the adoptive transfer of these modified Tregs would be an attractive strategy to prevent or treat Graft vs Host Disease (GVHD) [111]. It has been established that LKB1 downregulation in Tregs in a GVHD pathological condition and this is significant for targeting LKB1-related pathways to treat GVHD. As demonstrated in that, JQ1 (BET bromodomain inhibitor) (Table 1) enhanced the expression of LKB1, ATG5, and LC3-II genes and resulted in the phosphorylation of AMPK, ULK1, and ATG14 in allografts. This phosphorylation process has been reported to prolong heart allograft survival and inhibits the release of inflammatory cytokines [112], which further supports the role of the LKB1 gene in graft survival. Metabolic manipulation to Tregs and its expansion protocol holds tremendous promise in maintaining Treg-based tolerance during inflammatory disorders. It has been reported that rapamycin (Table 1) causes enhanced cell expansion and Treg stability [113]. In addition, recent progress in chimeric antigen receptors (CARs) technology affects the metabolic properties of Tregs [114-116] and enhance the specificity and functionality of Tregs $[35,117,118]$. It is an indirect strategy to modulate metabolic pathways in a celltype-specific manner. Thus, future studies should emphasize to use a combination of antimetabolites with tolerance-inducing regimens such as co-stimulatory blockade or LKB1 pathway augmentation or modified Treg adoptive transfer to provide specific and effective long-term graft acceptance.

\section{CONCLUSION:}

The role of immune signaling networks in immunometabolism is emerging as a promising area of research, which could provide a valuable tool to redesign therapeutic options to contain allograft rejection and the progression of chronic fibrosis. Here, we have discussed the critical roles of $\mathrm{T}$ cell's energy generation, roles in immunomodulation, and the impact of immunomodulation on transplantation. Altogether, the power of these metabolic signals contributes to the differentiation of Teffs; therefore, our understanding of the metabolic disparity among T-cell populations would open up new avenues to design therapeutic strategies to prevent inflammatory-related diseases.

\section{LIST OF ABBREVIATIONS:}

Teffs: T effector cells; Tregs: T regulatory cells; Tmem: T memory cells; APCs: Antigen-presenting cells; 
LKB1: Liver kinase B1; TCA: Tricarboxylic acid cycle; ETC: Electron transport chain; GLUT1: Glucose transporter 1; ATP: Adenosine triphosphate; NAD: Nicotinamide adenine dinucleotide; CoA: Coenzyme A; OXPHOS: Oxidative phosphorylation; LPS: Lipopolysaccharide; DC: Dendritic cell; NK: Natural killer; PRRs: Pattern Recognition receptor; FAO: Fatty acid oxidation; IL: Interleukin; CD: Cluster of differentiation; Th: T helper cells; CPT1A: Carnitine Palmitoyltransferase 1A; PD1: Programmed cell death 1; nTreg: natural Treg; iTreg: induced Treg; pTreg: peripheral Treg; PDH: pyruvate dehydrogenase; PDHKs: PDH kinases; NAC: N-acetyl cysteine; GCN2: general control nonderepressible-2; ARG1: arginase 1; HDC: histidine decarboxylase; TDH: threonine dehydrogenase; IL4I1: interleukin-4 induced 1; LDH: lactate dehydrogenase; mTOR: mammalian target of rapamycin; TIGIT: T cell immunoreceptor with immunoglobulin and ITIM domains; LAG3: lymphocyte activation gene 3; NRP1: neuropilin 1; CTLA4: cytotoxic T lymphocyte antigen 4; AMPK: AMP-activated protein kinase; ROS: reactive oxygen species; GITR: Glucocorticoid induced TNF receptor; HIF1- $\alpha$ : Hypoxia inducible factor 1- $\alpha \cdot$ mTORC1: mammalian target of rapamycin complex 1; IRI: Ischemaic reperfusion injury; aGVHD: acute graft- versus- host disease; CNS2: conserved non-coding sequences; STAT4: signal transducer and activator of transcription 4; NF- $\varkappa \mathrm{B}$ : nuclear factor- $\varkappa \mathrm{B}$; TGF- $\beta$ : transforming growth factor- $\beta$. GGPP: geranyl pyrophosphate; $2 D G$ : 2 -Deoxy-D-glucose; DON: 6Diazo-5-oxo-L-norleucine; ATG5: Autophagy related 5; LC3-II: light chain 3-II; ULK1: unc-51-like kinase 1; ATG14: Autophagy Related 14; BET: Bromodomain and Extra-Terminal motif; PPP: Pentose phosphate pathway; SRC: spare respiratory capacity.

Consent for publication: As per journal rules, I disclose that the work has not been published or submitted for publication elsewhere, either completely or in part, or another form or language

Availability of data and material: Not applicable

\section{ACKNOWLEDGEMENTS}

The author thanks all research staff of the organ transplant research center at King Faisal Special Hospital and Research Centre.

\section{Funding: Not Available}

Contributions: SK planned, and wrote the manuscript, MAK has been involved in writing, and revising it critically for publication standards. TS and AA contributed to various editing work. AS and DB provided the theme of the proposed research plan.

Corresponding author: Correspondence to Dr. Shadab Kazmi

ETHICS DECLARATION

Ethics approval and consent to participate: Not applicable.

Consent for publication: Not applicable.

\section{Competing interests: The author declares that they have no competing interests.}

\section{References}

1. Pearce EL, Poffenberger MC, Chang CH, Jones RG. Fueling immunity: insights into metabolism and lymphocyte function. Science. 2013 Oct 11;342(6155).

2. Gerriets VA, Kishton RJ, Nichols AG, Macintyre AN, Inoue M, Ilkayeva O, Winter PS, Liu X, Priyadharshini B, Slawinska ME, Haeberli L. Metabolic programming and PDHK1 control CD4+ T cell subsets and inflammation. The Journal of clinical investigation. 2015 Jan 2;125(1):194-207. 
3. Man K, Kallies A. Synchronizing transcriptional control of T cell metabolism and function. Nature reviews Immunology. 2015 Sep;15(9):574-84.

4. Ganeshan K, Chawla A. Metabolic regulation of immune responses. Annual review of immunology. 2014 Mar 21;32:609-34.

5. Fox CJ, Hammerman PS, Thompson CB. Fuel feeds function: energy metabolism and the T-cell response. Nature Reviews Immunology. 2005 Nov;5(11):844-52.

6. Palm W, Thompson CB. Nutrient acquisition strategies of mammalian cells. Nature. 2017 Jun;546(7657):234-42..

7. Lochner M, Berod L, Sparwasser T. Fatty acid metabolism in the regulation of T cell function. Trends in immunology. 2015 Feb 1;36(2):81-91..

8. Phan AT, Goldrath AW, Glass CK. Metabolic and epigenetic coordination of T cell and macrophage immunity. Immunity. 2017 May 16;46(5):714-29..

9. Loftus RM, Finlay DK. Immunometabolism: cellular metabolism turns immune regulator. Journal of Biological Chemistry. 2016 Jan 1;291(1):1-0.

10. Deng D, Xu C, Sun P, Wu J, Yan C, Hu M, Yan N. Crystal structure of the human glucose transporter GLUT1. Nature. 2014 Jun;510(7503):121-5.

11. Kaelin WG, Thompson CB. Clues from cell metabolism. Nature. 2010 Jun;465(7298):562-4.

12. Bolaños JP, Almeida A, Moncada S. Glycolysis: a bioenergetic or a survival pathway?. Trends in biochemical sciences. 2010 Mar 1;35(3):145-9.

13. Akram M. Citric acid cycle and role of its intermediates in metabolism. Cell biochemistry and biophysics. 2014 Apr 1;68(3):475-8.

14. Birsoy K, Wang T, Chen WW, Freinkman E, Abu-Remaileh M, Sabatini DM. An essential role of the mitochondrial electron transport chain in cell proliferation is to enable aspartate synthesis. Cell. 2015 Jul 30;162(3):540-51.

15. Kerner J, Hoppel C. Fatty acid import into mitochondria. Biochimica et Biophysica Acta (BBA)Molecular and Cell Biology of Lipids. 2000 Jun 26;1486(1):1-7.

16. Rodríguez-Prados JC, Través PG, Cuenca J, Rico D, Aragonés J, Martín-Sanz P, Cascante M, Boscá L. Substrate fate in activated macrophages: a comparison between innate, classic, and alternative activation. The Journal of Immunology. 2010 Jul 1;185(1):605-14.

17. Krawczyk CM, Holowka T, Sun J, Blagih J, Amiel E, DeBerardinis RJ, Cross JR, Jung E, Thompson $\mathrm{CB}$, Jones RG, Pearce EJ. Toll-like receptor-induced changes in glycolytic metabolism regulate dendritic cell activation. Blood. 2010 Jun 10;115(23):4742-9.

18. Donnelly RP, Loftus RM, Keating SE, Liou KT, Biron CA, Gardiner CM, Finlay DK. mTORC1dependent metabolic reprogramming is a prerequisite for NK cell effector function. The Journal of Immunology. 2014 Nov 1;193(9):4477-84.

19. Michalek RD, Gerriets VA, Jacobs SR, Macintyre AN, MacIver NJ, Mason EF, Sullivan SA, Nichols AG, Rathmell JC. Cutting edge: distinct glycolytic and lipid oxidative metabolic programs are essential for effector and regulatory CD4+ T cell subsets. The Journal of Immunology. 2011 Mar 15;186(6):3299-303.

20. Doughty CA, Bleiman BF, Wagner DJ, Dufort FJ, Mataraza JM, Roberts MF, Chiles TC. Antigen receptor-mediated changes in glucose metabolism in B lymphocytes: role of phosphatidylinositol 3-kinase signaling in the glycolytic control of growth. Blood. 2006 Jun 1;107(11):4458-65. 
21. Menk AV, Scharping NE, Moreci RS, Zeng X, Guy C, Salvatore S, Bae H, Xie J, Young HA, Wendell SG, Delgoffe GM. Early TCR signaling induces rapid aerobic glycolysis enabling distinct acute T cell effector functions. Cell reports. 2018 Feb 6;22(6):1509-21.

22. Saha S, Shalova IN, Biswas SK. Metabolic regulation of macrophage phenotype and function. Immunological Reviews. 2017 Nov;280(1):102-11.

23. Dimeloe S, Burgener AV, Grählert J, Hess C. T-cell metabolism governing activation, proliferation and differentiation; a modular view. Immunology. 2017 Jan;150(1):35-44.

24. Shimobayashi M, Hall MN. Multiple amino acid sensing inputs to mTORC1. Cell research. 2016 Jan;26(1):7-20.

25. Pacold ME, Brimacombe KR, Chan SH, Rohde JM, Lewis CA, Swier LJ, Possemato R, Chen WW, Sullivan LB, Fiske BP, Cho S. A PHGDH inhibitor reveals coordination of serine synthesis and one-carbon unit fate. Nature chemical biology. 2016 Jun;12(6):452-8.

26. Chang CH, Pearce EL. Emerging concepts of T cell metabolism as a target of immunotherapy. Nature immunology. 2016 Apr;17(4):364-8.

27. Almeida L, Lochner M, Berod L, Sparwasser T. Metabolic pathways in T cell activation and lineage differentiation. InSeminars in immunology 2016 Oct 1 (Vol. 28, No. 5, pp. 514-524). Academic Press.

28. Newton R, Priyadharshini B, Turka LA. Immunometabolism of regulatory T cells. Nature immunology. 2016 Jun;17(6):618.

29. van der Windt GJ, Everts B, Chang CH, Curtis JD, Freitas TC, Amiel E, Pearce EJ, Pearce EL. Mitochondrial respiratory capacity is a critical regulator of CD8+ T cell memory development. Immunity. 2012 Jan 27;36(1):68-78.

30. Cretenet G, Clerc I, Matias M, Loisel S, Craveiro M, Oburoglu L, Kinet S, Mongellaz C, Dardalhon V, Taylor N. Cell surface Glut1 levels distinguish human CD4 and CD8 T lymphocyte subsets with distinct effector functions. Scientific reports. 2016 Apr 12;6:24129.

31. Dumitru C, Kabat AM, Maloy KJ. Metabolic adaptations of CD4+ T cells in inflammatory disease. Frontiers in Immunology. 2018 Mar 15;9:540.

32. Procaccini C, Carbone F, Di Silvestre D, Brambilla F, De Rosa V, Galgani M, Faicchia D, Marone G, Tramontano D, Corona M, Alviggi C. The proteomic landscape of human ex vivo regulatory and conventional T cells reveals specific metabolic requirements. Immunity. 2016 Feb 16;44(2):406-21.

33. Bachem A, Makhlouf C, Binger KJ, de Souza DP, Tull D, Hochheiser K, Whitney PG, Fernandez-Ruiz D, Dahling S, Kastenmuller W, Jonsson J. Microbiota-derived short-chain fatty acids promote the memory potential of antigen-activated CD8+ T cells. Immunity. 2019 Aug 20;51(2):285-97.

34. Field CS, Baixauli F, Kyle RL, Puleston DJ, Cameron AM, Sanin DE, Hippen KL, Loschi M, Thangavelu G, Corrado M, Edwards-Hicks J. Mitochondrial integrity regulated by lipid metabolism is a cell-intrinsic checkpoint for Treg suppressive function. Cell metabolism. 2020 Feb 4;31(2):422-37.

35. Patsoukis N, Bardhan K, Chatterjee P, Sari D, Liu B, Bell LN, Karoly ED, Freeman GJ, Petkova V, Seth P, Li L. PD-1 alters T-cell metabolic reprogramming by inhibiting glycolysis and promoting lipolysis and fatty acid oxidation. Nature communications. 2015 Mar 26;6(1):1-3.

36. Piconese S, Pacella I. Immunometabolic checkpoints of Treg dynamics: adaptation to microenvironmental opportunities and challenges. Frontiers in immunology. 2019;10:1889.

37. Matarese G, Colamatteo A, De Rosa V. Metabolic fuelling of proper T cell functions. Immunology Letters. 2014 Oct 1;161(2):174-8. 
38. Bhattacharyya ND, Feng CG. Regulation of T helper cell fate by TCR signal strength. Frontiers in Immunology. 2020;11.

39. Morel PA. Differential T-cell receptor signals for T helper cell programming. Immunology. 2018 Sep;155(1):63-71.

40. Khan MA. T regulatory cell-mediated immunotherapy for solid organ transplantation: a clinical perspective. Molecular Medicine. 2016 Jan;22(1):892-904.

41. Khan MA, Alanazi F, Ahmed HA, Al-Mohanna FH, Assiri AM, Broering DC. FOXP3+ regulatory $\mathrm{T}$ cell ameliorates microvasculature in the rejection of mouse orthotopic tracheal transplants. Clinical Immunology. 2017 Jan 1;174:84-98.

42. Khan MA, Alanazi F, Ahmed HA, Shamma T, Kelly K, Hammad MA, Alawad AO, Assiri AM, Broering DC. iPSC-derived MSC therapy induces immune tolerance and supports long-term graft survival in mouse orthotopic tracheal transplants. Stem Cell Research \& Therapy. 2019 Dec;10(1):1-5.

43. Khan MA, Alanazi F, Ahmed HA, Vater A, Assiri AM, Broering DC. C5a blockade increases regulatory $\mathrm{T}$ cell numbers and protects against microvascular loss and epithelial damage in mouse airway allografts. Frontiers in immunology. 2018 May 24;9:1010.

44. Khan MA, Moeez S, Akhtar S. T-regulatory cell-mediated immune tolerance as a potential immunotherapeutic strategy to facilitate graft survival. Blood Transfusion. 2013 Jul;11(3):357.

45. Campbell DJ, Koch MA. Phenotypical and functional specialization of FOXP3+ regulatory T cells. Nature Reviews Immunology. 2011 Feb;11(2):119-30.

46. Shevach EM, Thornton AM. tTregs, pTregs, and iTregs: similarities and differences. Immunological reviews. 2014 May;259(1):88-102.

47. Sawant DV, Vignali DA. Once a Treg, always a Treg?. Immunological reviews. 2014 May;259(1):173-

91.

48. Mueller DL. Mechanisms maintaining peripheral tolerance. Nature immunology. 2010 Jan;11(1):21-7.

49. Verbist KC, Wang R, Green DR. T cell metabolism and the immune response. InSeminars in immunology 2012 Dec 1 (Vol. 24, No. 6, pp. 399-404). Academic Press.

50. Koppenol WH, Bounds PL, Dang CV. Otto Warburg's contributions to current concepts of cancer metabolism. Nature Reviews Cancer. 2011 May;11(5):325-37.

51. Wahl DR, Byersdorfer CA, Ferrara JL, Opipari Jr AW, Glick GD. Distinct metabolic programs in activated T cells: opportunities for selective immunomodulation. Immunological reviews. 2012 Sep;249(1):104-15..

52. Jacobs SR, Herman CE, MacIver NJ, Wofford JA, Wieman HL, Hammen JJ, Rathmell JC. Glucose uptake is limiting in T cell activation and requires CD28-mediated Akt-dependent and independent pathways. The Journal of Immunology. 2008 Apr 1;180(7):4476-86.

53. Maciolek JA, Pasternak JA, Wilson HL. Metabolism of activated T lymphocytes. Current opinion in immunology. 2014 Apr 1;27:60-74.

54. Galgani M, De Rosa V, La Cava A, Matarese G. Role of metabolism in the immunobiology of regulatory T cells. The Journal of Immunology. 2016 Oct 1;197(7):2567-75.

55. Kishore M, Cheung KC, Fu H, Bonacina F, Wang G, Coe D, Ward EJ, Colamatteo A, Jangani M, Baragetti A, Matarese G. Regulatory T cell migration is dependent on glucokinase-mediated glycolysis. Immunity. 2017 Nov 21;47(5):875-89. 
56. Howie D, Cobbold SP, Adams E, Ten Bokum A, Necula AS, Zhang W, Huang H, Roberts DJ, Thomas B, Hester SS, Vaux DJ. Foxp3 drives oxidative phosphorylation and protection from lipotoxicity. JCI insight. 2017 Feb 9;2(3).

57. Pacella I, Procaccini C, Focaccetti C, Miacci S, Timperi E, Faicchia D, Severa M, Rizzo F, Coccia EM, Bonacina F, Mitro N. Fatty acid metabolism complements glycolysis in the selective regulatory T cell expansion during tumor growth. Proceedings of the National Academy of Sciences. 2018 Jul 10;115(28):E6546-55.

58. Golias T, Papandreou I, Sun R, Kumar B, Brown NV, Swanson BJ, Pai R, Jaitin D, Le QT, Teknos TN, Denko NC. Hypoxic repression of pyruvate dehydrogenase activity is necessary for metabolic reprogramming and growth of model tumours. Scientific reports. 2016 Aug 8;6(1):1-1.

59. Shen H, Shi LZ. Metabolic regulation of TH17 cells. Molecular immunology. 2019 May 1;109:81-7.

60. Sun L, Fu J, Zhou Y. Metabolism controls the balance of Th17/T-regulatory cells. Frontiers in Immunology. 2017 Nov 27;8:1632.

61. Gerriets VA, Kishton RJ, Nichols AG, Macintyre AN, Inoue M, Ilkayeva O, Winter PS, Liu X, Priyadharshini B, Slawinska ME, Haeberli L. Metabolic programming and PDHK1 control CD4+ T cell subsets and inflammation. The Journal of clinical investigation. 2015 Jan 2;125(1):194-207.

62. McDonnell E, Crown SB, Fox DB, Kitir B, Ilkayeva OR, Olsen CA, Grimsrud PA, Hirschey MD. Lipids reprogram metabolism to become a major carbon source for histone acetylation. Cell reports. 2016 Nov 1;17(6):1463-72.

63. Deng G, Song X, Fujimoto S, Piccirillo CA, Nagai Y, Greene MI. Foxp3 post-translational modifications and Treg suppressive activity. Frontiers in immunology. 2019;10:2486.

64. Shi H, Chi H. Metabolic control of Treg cell stability, plasticity, and tissue-specific heterogeneity. Frontiers in Immunology. 2019;10.

65. van Loosdregt J, Coffer PJ. Post-translational modification networks regulating FOXP3 function. Trends in immunology. 2014 Aug 1;35(8):368-78.

66. Zeng H, Yang K, Cloer C, Neale G, Vogel P, Chi H. mTORC1 couples immune signals and metabolic programming to establish T reg-cell function. Nature. 2013 Jul;499(7459):485-90.

67. Carr EL, Kelman A, Wu GS, Gopaul R, Senkevitch E, Aghvanyan A, Turay AM, Frauwirth KA. Glutamine uptake and metabolism are coordinately regulated by ERK/MAPK during T lymphocyte activation. The Journal of Immunology. 2010 Jul 15;185(2):1037-44.

68. Lian G, Gnanaprakasam JR, Wang T, Wu R, Chen X, Liu L, Shen Y, Yang M, Yang J, Chen Y, Vasiliou V. Glutathione de novo synthesis but not recycling process coordinates with glutamine catabolism to control redox homeostasis and directs murine T cell differentiation. elife. 2018 Sep 10;7:e36158.

69. Sinclair LV, Rolf J, Emslie E, Shi YB, Taylor PM, Cantrell DA. Control of amino-acid transport by antigen receptors coordinates the metabolic reprogramming essential for $\mathrm{T}$ cell differentiation. Nature immunology. 2013 May;14(5):500-8.

70. Nakaya M, Xiao Y, Zhou X, Chang JH, Chang M, Cheng X, Blonska M, Lin X, Sun SC. Inflammatory T cell responses rely on amino acid transporter ASCT2 facilitation of glutamine uptake and mTORC1 kinase activation. Immunity. 2014 May 15;40(5):692-705.

71. Kolodziej LR, Paleolog EM, Williams RO. Kynurenine metabolism in health and disease. Amino acids. 2011 Nov 1;41(5):1173-83.

72. Mezrich JD, Fechner JH, Zhang X, Johnson BP, Burlingham WJ, Bradfield CA. An interaction between kynurenine and the aryl hydrocarbon receptor can generate regulatory $\mathrm{T}$ cells. The Journal of 
Immunology. 2010 Sep 15;185(6):3190-8.

73. Buck MD, O'sullivan D, Pearce EL. T cell metabolism drives immunity. Journal of Experimental Medicine. 2015 Aug 24;212(9):1345-60.

74. Priyadharshini B, Turka LA. T Cell Energy Metabolism as a Controller of Cell Fate in Transplantation. Current opinion in organ transplantation. 2015 Feb;20(1):21.

75. Kelly B, Pearce EL. Amino assets: how amino acids support immunity. Cell Metabolism. 2020 Jul 9.

76. Angelin A, Gil-de-Gomez L, Dahiya S, Jiao J, Guo L, Levine MH, Wang Z, Quinn III WJ, Kopinski PK, Wang L, Akimova T. Foxp3 reprograms T cell metabolism to function in low-glucose, high-lactate environments. Cell metabolism. 2017 Jun 6;25(6):1282-93.

77. Gerriets VA, Kishton RJ, Johnson MO, Cohen S, Siska PJ, Nichols AG, Warmoes MO, de Cubas AA, MacIver NJ, Locasale JW, Turka LA. Foxp3 and Toll-like receptor signaling balance T reg cell anabolic metabolism for suppression. Nature immunology. 2016 Dec;17(12):1459-66.

78. Lucca LE, Dominguez-Villar M. Modulation of regulatory T cell function and stability by co-inhibitory receptors. Nature Reviews Immunology. 2020 Apr 8:1-4.

79. Gurumurthy S, Xie SZ, Alagesan B, Kim J, Yusuf RZ, Saez B, Tzatsos A, Ozsolak F, Milos P, Ferrari F, Park PJ. The Lkb1 metabolic sensor maintains haematopoietic stem cell survival. Nature. 2010 Dec;468(7324):659-63.

80. Wu D, Luo Y, Guo W, Niu Q, Xue T, Yang F, Sun X, Chen S, Liu Y, Liu J, Sun Z. Lkb1 maintains T reg cell lineage identity. Nature communications. 2017 Jun 16;8(1):1-4.

81. Baas AF, Kuipers J, van der Wel NN, Batlle E, Koerten HK, Peters PJ, Clevers HC. Complete polarization of single intestinal epithelial cells upon activation of LKB1 by STRAD. Cell. 2004 Feb 6;116(3):457-66.

82. Yoo LI, Chung DC, Yuan J. LKB1 - a master tumour suppressor of the small intestine and beyond. Nature Reviews Cancer. 2002 Jul;2(7):529-35.

83. Shackelford DB, Shaw RJ. The LKB1-AMPK pathway: metabolism and growth control in tumour suppression. Nature Reviews Cancer. 2009 Aug;9(8):563-75.

84. MacIver NJ, Blagih J, Saucillo DC, Tonelli L, Griss T, Rathmell JC, Jones RG. The liver kinase B1 is a central regulator of T cell development, activation, and metabolism. The Journal of Immunology. 2011 Oct 15;187(8):4187-98.

85. Yang K, Blanco DB, Neale G, Vogel P, Avila J, Clish CB, Wu C, Shrestha S, Rankin S, Long L, Anil KC. Homeostatic control of metabolic and functional fitness of T reg cells by LKB1 signalling. Nature. 2017 Aug;548(7669):602-6.

86. He N, Fan W, Henriquez B, Ruth TY, Atkins AR, Liddle C, Zheng Y, Downes M, Evans RM. Metabolic control of regulatory $\mathrm{T}$ cell (Treg) survival and function by Lkb1. Proceedings of the National Academy of Sciences. 2017 Nov 21;114(47):12542-7.

87. Timilshina M, You Z, Lacher SM, Acharya S, Jiang L, Kang Y, Kim JA, Chang HW, Kim KJ, Park B, Song JH. Activation of mevalonate pathway via LKB1 is essential for stability of Treg cells. Cell reports. 2019 Jun 4;27(10):2948-61.

88. Chen S, Fang L, Guo W, Zhou Y, Yu G, Li W, Dong K, Liu J, Luo Y, Wang B, Li Z. Control of T reg cell homeostasis and immune equilibrium by Lkb1 in dendritic cells. Nature communications. 2018 Dec $13 ; 9(1): 1-6$. 
89. Fu Z, Ye J, Dean JW, Bostick JW, Weinberg SE, Xiong L, Oliff KN, Chen ZE, Avram D, Chandel NS, Zhou L. Requirement of mitochondrial transcription factor A in tissue-resident regulatory $\mathrm{T}$ cell maintenance and function. Cell reports. 2019 Jul 2;28(1):159-71.

90. Pelgrom LR, Patente TA, Sergushichev A, Esaulova E, Otto F, Ozir-Fazalalikhan A, van der Zande HJ, van der Ham AJ, van der Stel S, Artyomov MN, Everts B. LKB1 expressed in dendritic cells governs the development and expansion of thymus-derived regulatory T cells. Cell research. 2019 May;29(5):406-19.

91. Han SH, Malaga-Dieguez L, Chinga F, Kang HM, Tao J, Reidy K, Susztak K. Deletion of Lkb1 in renal tubular epithelial cells leads to CKD by altering metabolism. Journal of the American Society of Nephrology. 2016 Feb 1;27(2):439-53.

92. Koyama S, Akbay EA, Li YY, Aref AR, Skoulidis F, Herter-Sprie GS, Buczkowski KA, Liu Y, Awad MM, Denning WL, Diao L. STK11/LKB1 deficiency promotes neutrophil recruitment and proinflammatory cytokine production to suppress T-cell activity in the lung tumor microenvironment. Cancer research. 2016 Mar 1;76(5):999-1008.

93. Parker SJ, Svensson RU, Divakaruni AS, Lefebvre AE, Murphy AN, Shaw RJ, Metallo CM. LKB1 promotes metabolic flexibility in response to energy stress. Metabolic engineering. 2017 Sep 1;43:208-17.

94. Cai J, Wang D, Zhang G, Guo X. The role of PD-1/PD-L1 Axis in Treg development and function: implications for Cancer immunotherapy. OncoTargets and therapy. 2019;12:8437.

95. Whang YM, Park SI, Trenary IA, Egnatchik RA, Fessel JP, Kaufman JM, Carbone DP, Young JD. LKB1 deficiency enhances sensitivity to energetic stress induced by erlotinib treatment in non-small-cell lung cancer (NSCLC) cells. Oncogene. 2016 Feb;35(7):856-66.

96. Su X, Wang Q, Guo W, Pei X, Niu Q, Liu M, Liu Y, Chen S, Feng S, He Y, Yang D. Loss of Lkb1 impairs Treg function and stability to aggravate graft-versus-host disease after bone marrow transplantation. Cellular \& Molecular Immunology. 2020 May;17(5):483-95.

97. Polansky JK, Kretschmer K, Freyer J, Floess S, Garbe A, Baron U, Olek S, Hamann A, von Boehmer H, Huehn J. DNA methylation controls Foxp3 gene expression. European journal of immunology. 2008 Jun;38(6):1654-63.

98. Shalev I, Liu H, Koscik C, Bartczak A, Javadi M, Wong KM, Maknojia A, He W, Liu MF, Diao J, Winter E. Targeted deletion of fgl2 leads to impaired regulatory $\mathrm{T}$ cell activity and development of autoimmune glomerulonephritis. The Journal of Immunology. 2008 Jan 1;180(1):249-60.

99. Ochando J, Ordikhani F, Boros P, Jordan S. The innate immune response to allotransplants: mechanisms and therapeutic potentials. Cellular \& molecular immunology. 2019 Apr;16(4):350-6.

100. Gatza E, Wahl DR, Opipari AW, Sundberg TB, Reddy P, Liu C, Glick GD, Ferrara JL. Manipulating the bioenergetics of alloreactive $\mathrm{T}$ cells causes their selective apoptosis and arrests graft-versus-host disease. Science translational medicine. 2011 Jan 26;3(67):67ra8-.

101. Lee CF, Lo YC, Cheng CH, Furtmuller GJ, Oh B, Andrade-Oliveira V, Thomas AG, Bowman CE, Slusher BS, Wolfgang MJ, Brandacher G. Preventing allograft rejection by targeting immune metabolism. Cell reports. 2015 Oct 27;13(4):760-70.

102. El-Mir MY, Nogueira V, Fontaine E, Averet N, Rigoulet M, Leverve X. Dimethylbiguanide inhibits cell respiration via an indirect effect targeted on the respiratory chain complex I. Journal of Biological Chemistry. 2000 Jan 7;275(1):223-8.

103. O'Sullivan D, Pearce EL. Targeting T cell metabolism for therapy. Trends in immunology. 2015 Feb $1 ; 36(2): 71-80$.

104. Wang L, Li JJ, Guo LY, Li P, Zhao Z, Zhou H, Di LJ. Molecular link between glucose and glutamine consumption in cancer cells mediated by CtBP and SIRT4. Oncogenesis. 2018 Mar 13;7(3):1-0. 
105. Leone RD, Zhao L, Englert JM, Sun IM, Oh MH, Sun IH, Arwood ML, Bettencourt IA, Patel $\mathrm{CH}$, Wen J, Tam A. Glutamine blockade induces divergent metabolic programs to overcome tumor immune evasion. Science. 2019 Nov 22;366(6468):1013-21.

106. Park CS, Bang BR, Kwon HS, Moon KA, Kim TB, Lee KY, Moon HB, Cho YS. Metformin reduces airway inflammation and remodeling via activation of AMP-activated protein kinase. Biochemical pharmacology. 2012 Dec 15;84(12):1660-70.

107. Gundewar, S., Calvert, J.W., Jha, S., Toedt-Pingel, I., Yong Ji, S., Nunez, D., Ramachandran, A., Anaya-Cisneros, M., Tian, R. and Lefer, D.J., 2009. Activation of AMP-activated protein kinase by metformin improves left ventricular function and survival in heart failure. Circulation research, 104(3), pp.403-411.

108. Cordonier EL, Jarecke SK, Hollinger FE, Zempleni J. Inhibition of acetyl-CoA carboxylases by soraphen A prevents lipid accumulation and adipocyte differentiation in 3T3-L1 cells. European journal of pharmacology. 2016 Jun 5;780:202-8.

109. Duan W, Ding Y, Yu X, Ma D, Yang B, Li Y, Huang L, Chen Z, Zheng J, Yang C. Metformin mitigates autoimmune insulitis by inhibiting Th1 and Th17 responses while promoting Treg production. American Journal of Translational Research. 2019;11(4):2393.

110. Issa F, Wood KJ. CD4+ regulatory T cells in solid organ transplantation. Current opinion in organ transplantation. 2010 Dec;15(6):757.

111. Singer BD, King LS. D’ Alessio FR. Regulatory T cells as immunotherapy. 2014;5:46.

112. Chen J, Miao X, Liu C, Liu B, Wu X, Kong D, Sun Q, Gong W. BET Protein Inhibition Prolongs Cardiac Transplant Survival via Enhanced Myocardial Autophagy. Transplantation. 2020 Nov 1;104(11):231726.

113. Safinia N, Vaikunthanathan T, Fraser H, Thirkell S, Lowe K, Blackmore L, Whitehouse G, MartinezLlordella M, Jassem W, Sanchez-Fueyo A, Lechler RI. Successful expansion of functional and stable regulatory T cells for immunotherapy in liver transplantation. Oncotarget. 2016 Feb 16;7(7):7563.

114. Beavis PA, Henderson MA, Giuffrida L, Mills JK, Sek K, Cross RS, Davenport AJ, John LB, Mardiana $\mathrm{S}$, Slaney CY, Johnstone RW. Targeting the adenosine 2A receptor enhances chimeric antigen receptor $\mathrm{T}$ cell efficacy. The Journal of clinical investigation. 2017 Mar 1;127(3):929-41.

115. Xu X, Gnanaprakasam JN, Sherman J, Wang R. A metabolism toolbox for CAR T therapy. Frontiers in oncology. 2019 Apr 30;9:322.

116. Li G, Boucher JC, Kotani H, Park K, Zhang Y, Shrestha B, Wang X, Guan L, Beatty N, Abate-Daga D, Davila ML. 4-1BB enhancement of CAR T function requires NF- $x$ B and TRAFs. JCI insight. 2018 Sep $20 ; 3(18)$.

117. Chen Y, Colello J, Jarjour W, Zheng SG. Cellular metabolic regulation in the differentiation and function of regulatory T cells. Cells. 2019 Feb;8(2):188.

118. Ferreira LM, Muller YD, Bluestone JA, Tang Q. Next-generation regulatory T cell therapy. Nature Reviews Drug Discovery. 2019 Oct;18(10):749-69.

\section{Figure legends and Tables}

Fig.1 Factors that influences metabolic phenotype of inflammatory and anti-inflammatory immune cells towards anti-inflammatory phenotype: The various immune cell subtype reliance on distinct metabolic pathways to promote their survival, lineage subtype and function in the presence of several factors. mTOR-HIF1- $\alpha$ signaling axis promote inflammatory macrophages (M1) and effector T cells (Th1,Th2,Th17) proliferation and function through utilizing glycolysis, fatty acid synthesis and amino acid 
metabolism as a main energy source. Contray to this, Tregs, Tmem and M2 macrophages, which show a more tolerant phenotype, use the TCA cycle and fatty acid oxidation for their energy source under the influence of LKB1 signaling. Figure created with Biorender.com

Fig.2 LKB1 and AMPK Signaling allows metabolic programming in T cells: Concerning with low nutrient and energy status, the energy stress pathway kinases LKB1 and AMPK are triggered through TCR and CD28 co-stimulatory signals, with AMPK activity mainly triggered by most understood Ca2+CAMMK2 (calcium calmodulin kinase kinase 2) pathway. Bioenergetic fluctuations in cells such as deprivation of glucose or glutamine or elevation of AMP/ADP-to-ATP ratio, can also activate LKB1-AMPK signaling. The LKB1 cellular localization and post-translational modifications play an important role in its activity. The role of many upstream regulators of LKB1 signaling in T cells metabolic programming is still undiscovered. The activation of LKB1 promote mitochondrial fitness and increased mevalonate metabolism in Treg cells. The downstream kinase AMPK is the best-known kinase of LKB1. LKB1 is a critical metabolic regulator that regulates energy homeostasis in Tregs. AMPK also promotes mitochondrial fitness by increasing mitochondrial mass. Figure created with Biorender.com

Fig.3 Strategies to modulate metabolism to regulate $T$ cell function in diseases: The figure provides a summary of strategies to pharmacologically target (green) metabolic pathways crucial for $\mathrm{T}$ cells responses (black). This figure depicts the utilization of three key metabolic pathways namely glycolysis, FAO and glutaminolysis for the treatment of experimental autoimmune encephalomyelitis (EAE), multiple sclerosis (MS), rheumatoid arthritis (RA), systemic lupus erythematosus (SLE), and transplantation. Figure created with Biorender.com

Table 1. Pharmacological inhibitors of metabolic pathways and their effects on Treg and in diseases

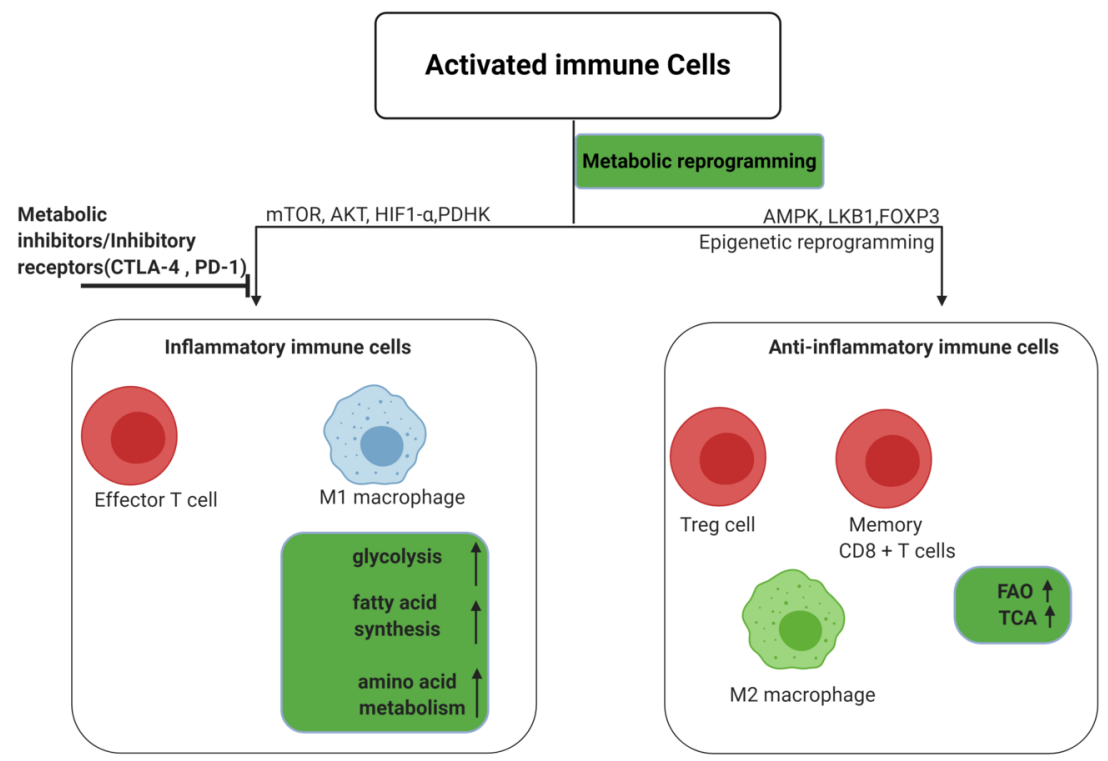

Figure 1: 
Figure 1. Factors that influences metabolic phenotype of inflammatory and anti-inflammatory immune cells towards anti-inflammatory phenotype

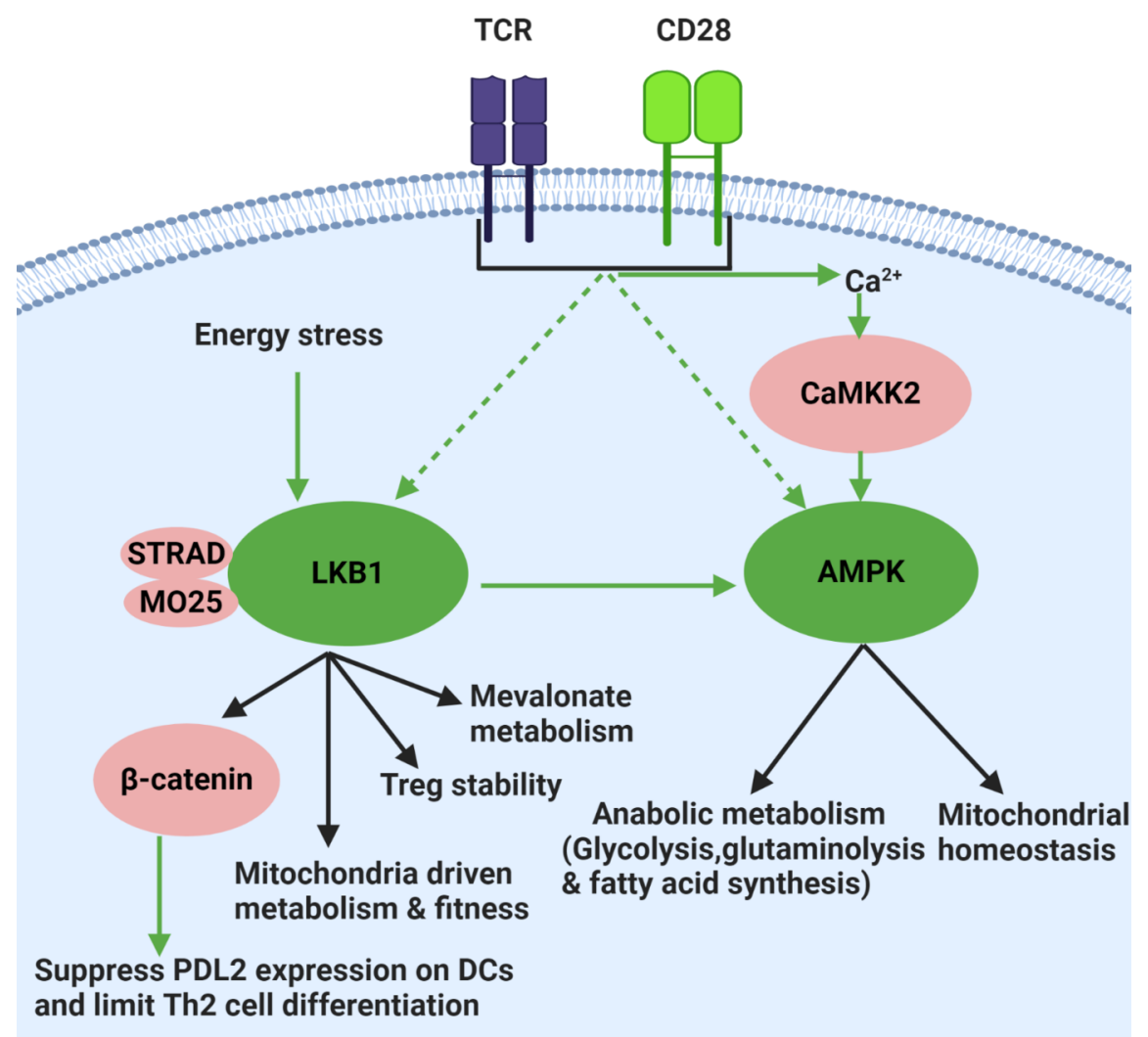

Figure 2. LKB1 and AMPK Signaling allows metabolic programming in T cells 


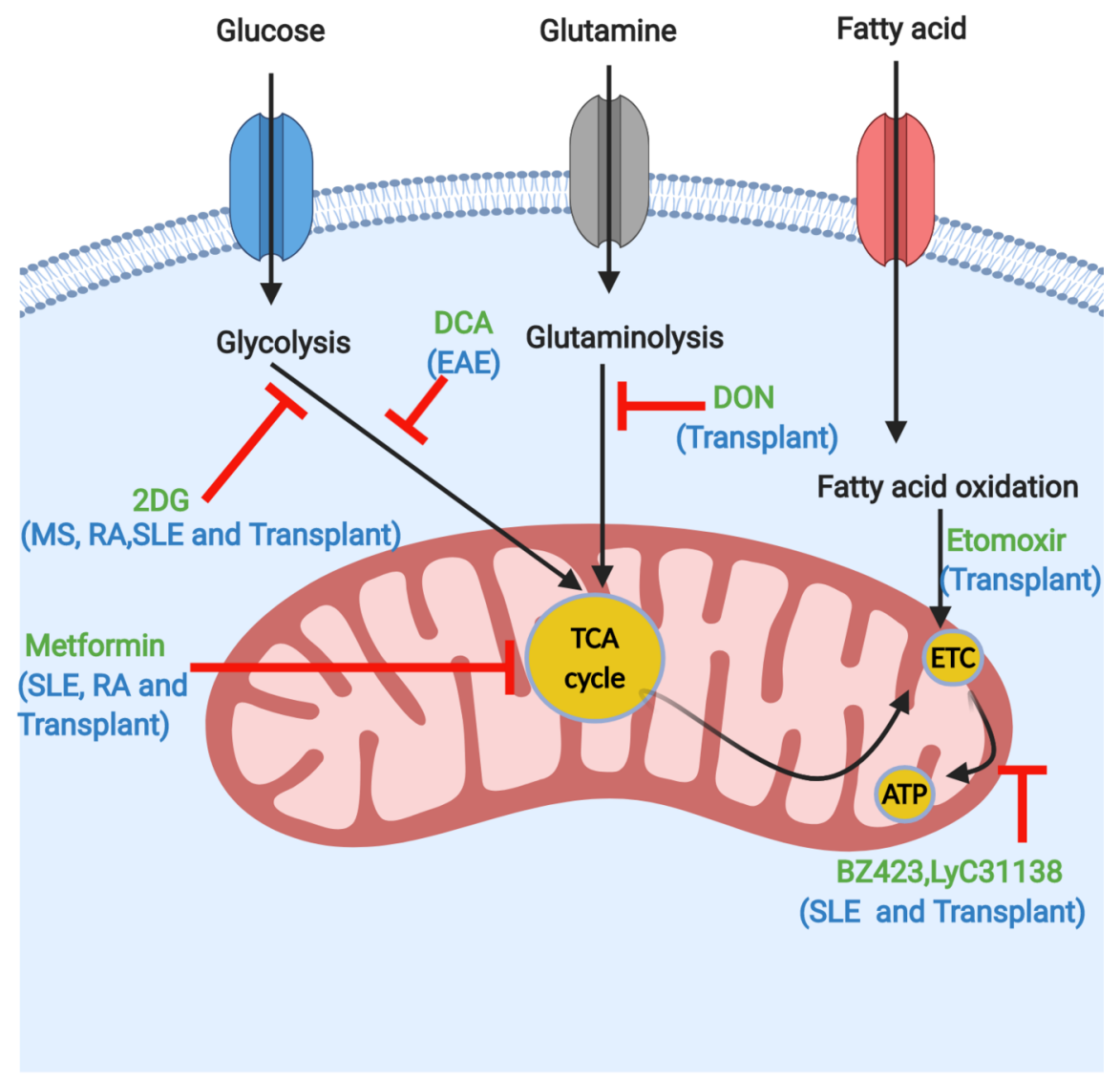

Figure 3. Strategies to modulate metabolism to regulate $\mathbf{T}$ cell function in diseases

Table 1. Pharmacological inhibitors of metabolic pathways and their effects on Treg and in diseases

\begin{tabular}{|c|c|c|c|c|}
\hline Metabolic Pathway & Drug & & Target & Effect on Tregs \\
\hline Glucose metabolism & $2-\mathrm{DG}$ & Dichloroacetate & Glycolysis $\quad$ PDHK & Induce Treg differentiation \\
\hline Lipid metabolism & Soraphen A & & acetyl-CoA carboxylase inhibitors & Induce pTreg differentiation \\
\hline Glutamine metabolism & DON & & Glutamine uptake & Induce Treg generation \\
\hline Metabolic regulators & Rapamycin & Metformin & mTORC1 AMPK & Enhance Treg cell expansion \\
\hline Epigenetic inhibtor & JQ1 & & BET bromodomain inhibitor & Enhanced expression of LKB \\
\hline
\end{tabular}


